\title{
Effect of magnetic disorder and strong electron correlations on the thermodynamics of $\mathrm{CrN}$
}

\author{
Björn Alling, Tobias Marten and Igor Abrikosov
}

\section{Linköping University Post Print}

N.B.: When citing this work, cite the original article.

Original Publication:

Björn Alling, Tobias Marten and Igor Abrikosov, Effect of magnetic disorder and strong electron correlations on the thermodynamics of CrN, 2010, Physical Review B. Condensed Matter and Materials Physics, (82), , 184430.

http://dx.doi.org/10.1103/PhysRevB.82.184430

Copyright: American Physical Society http://www.aps.org/

Postprint available at: Linköping University Electronic Press http://urn.kb.se/resolve?urn=urn:nbn:se:liu:diva-60439 


\title{
Effect of magnetic disorder and strong electron correlations on the thermodynamics of $\mathrm{CrN}$
}

\author{
B. Alling, ${ }^{*}$ T. Marten, and I. A. Abrikosov \\ Department of Physics, Chemistry and Biology, IFM, Linköping University, SE-581 83 Linköping, Sweden
}

(Received 12 June 2010; published 29 November 2010)

\begin{abstract}
We use first-principles calculations to study the effect of magnetic disorder and electron correlations on the structural and thermodynamic properties of $\mathrm{CrN}$. We illustrate the usability of a special quasirandom structure supercell treatment of the magnetic disorder by comparing with coherent potential approximation calculations and with a complementary magnetic sampling method. The need of a treatment of electron correlations effects beyond the local density approximation is proven by a comparison of LDA $+U$ calculations of structural and electronic properties with experimental results. When magnetic disorder and strong electron correlations are taken into account simultaneously, pressure- and temperature-induced structural and magnetic transitions in $\mathrm{CrN}$ can be understood.
\end{abstract}

DOI: $10.1103 /$ PhysRevB.82.184430

PACS number(s): 75.10.-b, 75.20.En, 75.20.Hr

\section{INTRODUCTION}

Transition-metal nitrides have attracted much interest due to their excellent performance in a long range of industrial application such as hard protective coatings on cutting tools, diffusion barriers, and wear resistant electrical contacts. $\mathrm{CrN}$ is not as hard as for instance TiN (Ref. 1) but it is superior to $\mathrm{TiN}$ in solving large concentrations of $\mathrm{AlN}$ in the rocksalt phase giving rise to the $\mathrm{Cr}_{1-x} \mathrm{Al}_{x} \mathrm{~N}$ solid solutions highly valued in hard-coatings applications. ${ }^{2-4}$ Furthermore, $\mathrm{CrN}$ on its own can be found in coating application for metal-forming and plastic-molding purposes. ${ }^{5,6}$ From a fundamental physics point of view the study of $\mathrm{CrN}$ has provided new insights but also raised questions about magneto-driven structural transitions. It is known that a magnetic order-disorder transition at temperatures around $280 \mathrm{~K}$ is associated with an orthorhombic to cubic structural transition, ${ }^{7}$ recently observed to be reversible under small pressures. ${ }^{8}$ On the other hand in epitaxially stabilized cubic thin films no sign of magnetic ordering has been seen. ${ }^{9-11}$ Recently Bhobe et al. ${ }^{12}$ highlighted the importance of electron correlations in $\mathrm{CrN}$ based on photoemission spectroscopy. On the theoretical side the concept of magnetic stress has been introduced and used within a local density approximation (LDA) framework to explain the orthorhombic distortion. ${ }^{13,14}$ More recently it was shown theoretically that taking strong electron correlations into account in the calculations at the level of the local spin-density approximation plus a Hubbard $U$-term $(\mathrm{LDA}+U)$ could improve the agreement between calculations and experiments by opening up a small band gap at the Fermi level. ${ }^{15}$ However, all these calculations considered only ordered magnetic structures while most experimental measurements, especially of the band structure, are performed above the Néel temperature.

Unlike what is sometimes assumed, most magnetic systems retain magnetic moments also above their critical Curie or Néel temperature. Indeed local moments are typically present although long-range order between them is lost. $\mathrm{CrN}$ is such a system where the experimentally observed structural (lattice spacing) and electronic properties (semiconducting behavior) of the paramagnetic cubic phase cannot be even qualitatively reproduced by nonmagnetic calculations. ${ }^{13}$
At the same time, when performing first-principles calculations modeling such disordered cases, ordered magnetic structures should not be used because they might give rise to order-specific features, like the well-known mixing anomaly in the $\mathrm{Fe}_{1-x} \mathrm{Cr}_{x}$ (Ref. 16) system. This means that a disordered magnetic state must be considered in order to fully understand the physics of paramagnetic $\mathrm{CrN}$ at room temperature.

For such a purpose the disordered local moments (DLM) (Ref. 17) method has been suggested and implemented within the coherent potential approximation (CPA) (Ref. 18) treatment for disorder. The DLM-CPA method was used in Ref. 19 to demonstrate the importance of the magnetic degree of freedom when paramagnetic cubic $\mathrm{CrN}$ was alloyed with AlN. Even though the DLM-CPA treatment of magnetic disorder is an excellent approximation in many cases, a direct method for calculating the electronic structure of magnetically disordered systems within a conventional supercell methodology is highly desirable. This is so since the CPA is most often combined with other approximations, e.g., the spherical approximation for the single-particle potential, imposing certain limitations on the treatment of materials with complex underlying crystal lattices. Moreover, in magnetic alloys, such as $\mathrm{Cr}_{1-x} \mathrm{Al}_{x} \mathrm{~N}$ or $\mathrm{Fe}_{1-x} \mathrm{Ni}_{x},{ }^{20,21}$ or in the presence of defects such as nitrogen vacancies in $\mathrm{CrN}$, local lattice relaxations and other local environment effects might be important and they are beyond the reach of the single-site CPA theory. Furthermore, if the magnetic and vibrational thermodynamics of solids are ever to be treated simultaneously on the same footing, a supercell treatment of magnetism, compatible with quantum molecular-dynamics simulations needs to be developed.

In this work we apply two different supercell approaches to treat the magnetic disorder of the paramagnetic phase of $\mathrm{CrN}$ and compare them with DLM-CPA calculations. First we apply the special quasirandom structure (SQS) method, ${ }^{22}$ developed to treat chemically disordered alloy systems. Second, to gain further confidence, we propose a magnetic sampling method (MSM) and show that the two methods give equivalent results.

Furthermore we investigate the impact of strong electron correlations on structural and electronic properties of $\mathrm{CrN}$ at the level of $\mathrm{LDA}+U$ calculations. Considering both mag- 
netic disorder and strong electron correlations simultaneously we analyze the magnetostructural transition in $\mathrm{CrN}$.

\section{CALCULATIONAL DETAILS}

In this work electronic structure calculations are performed within a density-functional-theory framework and the projector augmented wave (PAW) method ${ }^{23}$ as implemented in the Vienna $a b$ initio simulation package (VASP). ${ }^{24,25}$ Both the LDA, ${ }^{26}$ the generalized gradient approximation (GGA) (Ref. 27) and a combination of the LDA with a Hubbard Coulomb term $(\mathrm{LDA}+U)$ (Refs. 28 and 29) methods are used for treating electron exchange-correlation effects. The Hubbard term is applied only to the $\mathrm{Cr} 3 d$ orbitals. In this implementation of the LDA $+U$ method, using the double-counting correction scheme according to Dudarev et al. ${ }^{29}$ there is only one free parameter corresponding to $U^{e f f}=(U-J)$. In the following the simple notation $U$ is used for $U^{e f f}$. The energy cutoff for plane waves included in the expansion of wave functions are $400 \mathrm{eV}$. Sampling of the Brillouin zone was done using a Monkhorst-Pack scheme ${ }^{30}$ on a grid of $5 \times 5 \times 5$ (64-atom supercells), $9 \times 9 \times 9$ (48atoms supercells), $13 \times 13 \times 13$ (8-atom cells), and $21 \times 21$ $\times 21$ (2-atoms cells) $k$ points. To find the optimal cell geometry for the orthorhombic structure an automatic optimization procedure was used independently for each volume. We also apply the exact muffin-tin orbitals (EMTO) method ${ }^{31,32}$ including the full charge-density technique ${ }^{33}$ in which the DLM-CPA treatment of magnetic disorder is implemented. The EMTO basis set included $s, p, d$, and $f$ orbitals, and the total energies were converged within $0.5 \mathrm{meV} / \mathrm{f}$.u. with respect to the density of the $k$-point mesh.

\section{SUPERCELL APPROACH TO MAGNETIC DISORDER}

\section{A. Energy of a disordered magnet}

In this work we discuss the thermodynamics of the hightemperature paramagnetic phase of $\mathrm{CrN}$. The modeling of such a state is a nontrivial many-body problem. This issue has been discussed in the literature, ${ }^{17,34}$ where it was shown that this state can be simulated within the DLM approach. Indeed, according to Ref. 34 the latter model gives a "static approximation" to the complete theory where charge and spin fields are dynamically fluctuating both in space and time. Though the dynamics of the fluctuations is neglected in the DLM picture, it does capture the important part of the correlations and is highly attractive for practical applications because it significantly simplifies the problem. This approach should be particularly suitable in the case of $\mathrm{CrN}$ due to the robust local moments ${ }^{19}$ and a half-filled $t_{2 g}$ band without any sign of a Kondo resonance. ${ }^{9}$ This model was also used with considerable success in practical applications. ${ }^{16,35,36}$

We will now try to establish at what conditions the DLM description, traditionally implemented within the CPA, can be extended toward a supercell technique, and therefore can be combined with very accurate full-potential treatments of the one-electron problem. We will base our discussion on the classical Heisenberg model with the Hamiltonian

$$
H_{m a g}=-\sum_{i \neq j} J_{i j} \mathbf{e}_{i} \cdot \mathbf{e}_{j}=-\sum_{\alpha} J_{\alpha} n_{\alpha}\left\langle\Phi_{\alpha}\right\rangle,
$$

where $J_{i j}$ are the interaction parameters, $\mathbf{e}_{i}$ is a unit vector in the direction of the magnetic moment on site $i\left(\mathbf{e}_{i}=\mathbf{S}_{i} / M\right.$, where $M$ is the magnitude of the magnetic moments), $\alpha$ corresponds to a specific coordination shell, $n_{\alpha}$ is the number of atoms in the $\alpha$ : th coordination shell on the lattice, and $\left\langle\Phi_{\alpha}\right\rangle$ is the spin-correlation functions to be defined below.

This model is consistent with the DLM approach and is usually giving a good description of the magnetism of itinerant electron systems when they display a Heisenberg-type behavior of the magnetic moments. Such a behavior is indeed present in $\mathrm{CrN}$ where the magnetic moments have been shown to be formed due to a magnetic split of the $\mathrm{Cr} t_{2}$ nonbonding $d$ states present at the Fermi level. ${ }^{13}$ The moments are large and they present stable values regardless if they are ordered in a ferromagnetic configuration, antiferromagnetic configuration, or a disordered local moments configuration. In this work, when using the GGA, we obtain $\mathrm{Cr}$ magnetic moments of $2.46 \mu_{B}$ in the ferromagnetic state, $2.37 \mu_{B}$ in the cubic single layer [001] ordered antiferromagnetic $\left(\mathrm{AFM}[001]_{1}\right)$ state, $2.41 \mu_{B}$ in the orthorhombic double layer [011] ordered antiferromagnetic (AFM [011] 2 ) state, and $2.49 \mu_{B}$ as the mean value in the DLM calculations. In the $\mathrm{LDA}+U$ calculations with $U=3 \mathrm{eV}$, the corresponding values are $2.96 \mu_{B}, 2.72 \mu_{B}, 2.81 \mu_{B}$, and $2.82 \mu_{B}$, respectively. Similar results were found in Ref. 19 . We note that the Heisenberg model, although limited to the first two nearest-neighbor interactions, was applied in Ref. 14 for analyzing magnetic-induced stress. However, one should be aware that it has been shown that the interaction parameters $J_{i j}$ could depend quite substantially on the global magnetic state even if the magnetic moments are almost constant, ${ }^{37}$ underlining the importance of a reliable method directly accessing the disordered magnetic state.

The paramagnetic state of $\mathrm{CrN}$ can within this model be described as a disordered distribution of $\mathrm{Cr}$ magnetic moments on the lattice, lacking long-range order. Similar situations are believed to be present in many other systems such as bec Fe and its alloys at high temperatures, ${ }^{35,38} \mathrm{NiMnSb}$ above the Curie temperature ${ }^{37}$ as well as a large number of $f$-electron systems. ${ }^{34}$ The next problem we encounter is thus how to calculate the energy of such a disordered magnet using a supercell technique.

In order to create an adequate supercell that can be used we need to know the characteristics of the random distribution. An ideally random distribution of magnetic spins, corresponding in the Heisenberg model [Eq. (1)] to infinite temperature, is characterized by the vanishing of all the average spin correlation functions

$$
\left\langle\Phi_{\alpha}\right\rangle=\frac{1}{N} \sum_{i, j \in \alpha} \mathbf{e}_{i} \cdot \mathbf{e}_{j}=0, \forall \alpha,
$$

where $N$ is a normalization constant.

Two important observations can be made from Eqs. (1) and (2). First, in order to calculate the total energy of the ideal random distribution, the structure we use in simulations has to fulfill the condition in Eq. (2) only for the coordina- 
tion shells $\alpha$ where the interaction parameters $J_{\alpha}$ are nonnegligible. Second, one realizes that even though the disordered spin state is utterly noncollinear, its energy can be calculated using a collinear state as long as the parallel $\left(\mathbf{S}_{i} \cdot \mathbf{S}_{j}=+1 M^{2}\right)$ and antiparallel $\left(\mathbf{S}_{i} \cdot \mathbf{S}_{j}=-1 M^{2}\right)$ spin pairs exactly cancel on each relevant coordination shell resulting in $\left\langle\widetilde{\Phi}_{\alpha}^{c o l}\right\rangle=0$. Thus, it is these characteristics that we should aim for in our simulation of the high-temperature paramagnetic state.

It is not directly obvious how to create a supercell fulfilling these properties but it is in fact a situation very similar to the problem of modeling chemical disorder in the form of a binary substitutional random alloy. ${ }^{39}$ The DLM-CPA method mentioned above can actually be seen as just a CPA treatment of a random alloy of atoms with spin-up and spin-down oriented magnetic moments. If we follow this analogy to the supercell framework it is the SQS methodology, first suggested by Zunger et $a l^{22}$ that has proven to be the most accurate approach for direct calculations of the total energy or related properties of the disordered state. The agreement between the CPA and SQS methods for chemical disorder in the $\mathrm{B} 1$ structure was demonstrated for the $\mathrm{Ti}_{1-x} \mathrm{Al}_{x} \mathrm{~N}$ system in Ref. 40 using the same electronic structure methods as in this work. Here we use the 48-atom $(24$ chromium +24 nitrogen) SQS structure suggested in Ref. 40 for $\mathrm{Ti}_{0.5} \mathrm{Al}_{0.5} \mathrm{~N}$ to model the $\mathrm{Cr}_{0.5}^{\uparrow} \mathrm{Cr}_{0.5}^{\downarrow} \mathrm{N}$ paramagnetic phase. That structure has $\left\langle\widetilde{\Phi}_{\alpha}^{c o l}\right\rangle=0$ for the first seven coordination shells with the exception of a small nonzero value on the fifth shell. For comparison reasons we also use a 64 -atom (32 chromium +32 nitrogen) SQS structure based on a cubic $2 \times 2 \times 2$ conventional unit cell geometry with $\left\langle\widetilde{\Phi}_{\alpha}^{c o l}\right\rangle=0$ for the first seven shells with the exception of small nonzero values on the third and seventh shells. We used the SQS method in a study of the bulk modulus of the paramagnetic phase of CrN (Ref. 41) as well as to get a $\mathrm{CrN}$ reference energy in a study of $\mathrm{Ti}_{1-x} \mathrm{Cr}_{x} \mathrm{~N} .{ }^{42}$ However the reliability of the method was questioned in Ref. 43.

To check if the SQS method is reliable to model magnetic disorder on the cubic lattice of $\mathrm{CrN}$ we show in Fig. 1 a comparison of energy-lattice parameter curves for different magnetic states including the disordered DLM state calculated with the SQS-PAW (left panel) and CPA-EMTO (right panel) methods employing the GGA functional for exchangecorrelation effects. The energies are given relative to the nonmagnetic energy minimum. The agreement between the two different treatments of disordered magnetism is clearly seen as the disordered state is placed in a very similar relation to ordered magnetic and nonmagnetic calculations in the two methodological frameworks.

Here we note that the accuracy of the DLM-CPA treatment of a completely disordered magnetic state is established analytically on the single-site level in Ref. 17. We therefore view very good agreement between SQS and DLM-CPA calculations as a strong proof that the former technique is capable to describe the energy of a paramagnetic state, at least at the same level of accuracy. In particular, it is clear that the SQS method does not suffer from imposed periodic boundary conditions or the fact that one technically speaking deals with one selected antiferromagnetic configuration. As soon

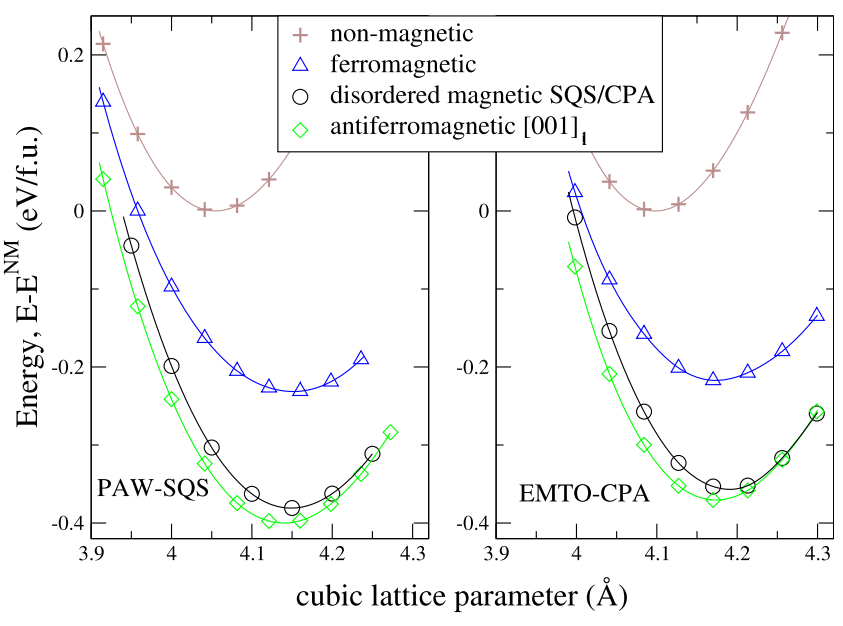

FIG. 1. (Color online) The energies as a function of lattice parameter of different magnetic states of cubic $\mathrm{B} 1 \mathrm{CrN}$ as calculated with the PAW and EMTO methods and the GGA exchangecorrelation functional. The disordered phase (circles) is modeled with the SQS method in the PAW calculations and the DLM-CPA method in the EMTO calculations.

as conditions given by Eqs. (2) and (1) are fulfilled, the SQS represents a quasirandom rather than ordered magnetic state. As for some minor differences between the results presented in the two panels of Fig. 1, they come from the usage of different underlying methods for the electronic-structure calculations, PAW and EMTO.

In order to further establish the reliability of the SQS approach, using the same PAW methodology, we first compare the results calculated for the two different SQS geometries considered in this work. The energy difference between them is $0.003 \mathrm{eV} / \mathrm{f} . \mathrm{u}$. Due to the translational symmetry the SQS based on the $2 \times 2 \times 2$ conventional unit cells has the problem that the correlation function on the eighth correlation shell is exactly 1 and this is probably the main source of the small difference between the two SQSs.

Next we suggest a different method to calculate the energy of a magnetic state approximating that in Eq. (2): the MSM. Within the MSM the directions (up and down) of magnetic moments of the $\mathrm{Cr}$ sites of a large supercell are chosen using a random number generator. A large set of different such distributions, magnetic samples, are then created. Their energies are calculated and the average energy is taken as the energy of the disordered state. Individually these supercells typically do not satisfy the conditions of Eqs. (1) and (2) but their average should, given that a sufficiently large number is considered. Although the SQS formalism was suggested in a reaction against the inaccuracies of random number distribution schemes, ${ }^{22}$ we note that the supercell sizes and particularly the number of calculations possible to treat with today's computational resources are orders of magnitude larger as compared to those back in 1990 .

Figure 2 shows the calculated energies of 40 different randomly generated magnetic samples with up and down collinear moments on the ideal lattice points of a $32 \mathrm{Cr}$ atoms $(2 \times 2 \times 2$ conventional unit cells $) \mathrm{B} 1 \mathrm{CrN}$ supercell. Their accumulated average energy is shown with a solid line and compared to the energy of the SQS generated configu- 


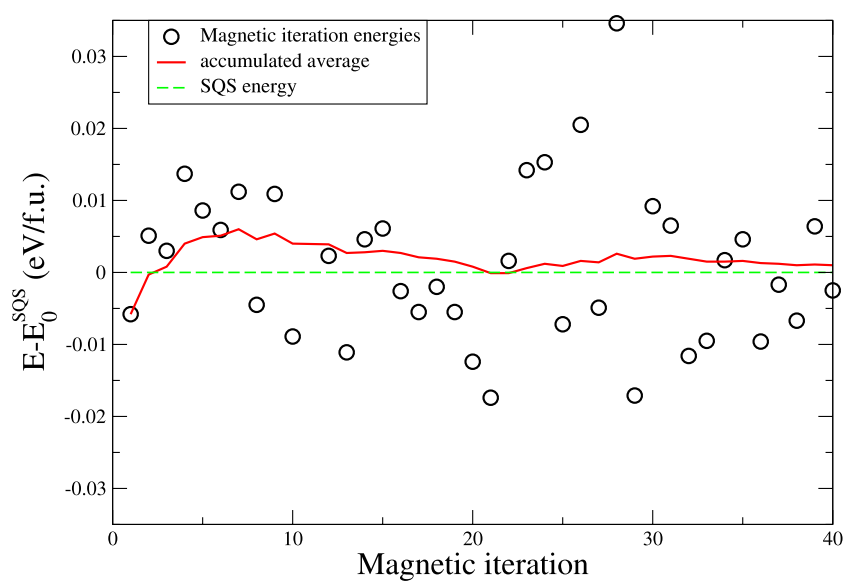

FIG. 2. (Color online) The calculated energies of the different random number generated magnetic configurations of a $32 \mathrm{Cr}-$ atoms $\mathrm{B} 1 \mathrm{CrN}$ supercell (circles). The accumulated average of the random number generated configurations, called magnetic iterations, is shown with a solid line. All values are plotted relative the energy minimum of a SQS-based magnetic configuration on the same underlying structure.

ration on the same underlying geometry. The latter is taken as the reference energy. Although the individual energies of the randomly generated supercells can differ as much as $-0.02 \mathrm{eV} /$ f.u. and $+0.035 \mathrm{eV} /$ f.u. from the SQS value, the accumulated average after the consideration of 40 different samples, called magnetic iterations, differs only by 0.001 eV/f.u. Between iteration 20 and 40, the average is never more than $0.001 \mathrm{eV} / \mathrm{f}$.u. above or $0.0015 \mathrm{eV} / \mathrm{f}$.u. below the value at iteration 40 showing that the mean energy is converging. If needed one could use even more sampled structures to converge the value with a higher accuracy than the 40 used in the present work. With this said we conclude, by our comparison with the DLM-CPA calculations as well as the internal agreement between SQS and MSM methods that both the considered supercell approaches can be used to calculate the total energy of a disordered collinear magnetic state of $\mathrm{CrN}$ with an accuracy of a few meV/f.u. on a fixed ideal B1 lattice.

\section{B. Noncollinear considerations}

Finally, we note that for systems where the energetics of the noncollinear disordered state is believed not to be well described by Eq. (1), for instance, due to non-negligible contributions from biquadratic terms in the Hamiltonian

$$
\begin{aligned}
H_{\text {mag }}= & -\sum_{i \neq j} J_{i j} \mathbf{e}_{i} \cdot \mathbf{e}_{j}-\sum_{i \neq j} K_{i j}\left(\mathbf{e}_{i} \cdot \mathbf{e}_{j}\right)^{2}=-\sum_{\alpha}\left(J_{\alpha} n_{\alpha}\left\langle\Phi_{\alpha}\right\rangle\right. \\
& \left.+K_{\alpha} n_{\alpha}\left\langle\Psi_{\alpha}\right\rangle\right),
\end{aligned}
$$

where in the fully disordered state

$$
\left\langle\Psi_{\alpha}\right\rangle=\frac{1}{N} \sum_{i, j \in \alpha}\left(\mathbf{e}_{i} \cdot \mathbf{e}_{j}\right)^{2}=\frac{1}{3}, \forall \alpha
$$

the MSM method could still be used with a straight forward generalization: Instead of the random number generation of collinear spins (up and down in $\hat{\mathbf{z}}$ ) which would give $\left\langle\widetilde{\Psi}_{\alpha}^{c o l}\right\rangle=1$, one can generate a set of different noncollinear supercells with six types of local moments describing up and down along $\hat{\mathbf{x}}, \hat{\mathbf{y}}$, and $\hat{\mathbf{z}}$. Such a noncollinear set, given that the supercells are large enough and that the number of supercells is large enough, will reproduce both the bilinear [Eq. (2)] $\left\langle\widetilde{\Phi}_{\alpha}^{n c}\right\rangle=0$, and biquadratic [Eq. (4)] $\left\langle\widetilde{\Psi}_{\alpha}^{n c}\right\rangle=\frac{1}{3}$, correlation functions of the disordered state. In principle, but cumbersome in practise, also the SQS method can be used in this case by constructing a large supercell of six components with vanishing correlation functions between them all.

Unfortunately, many electronic-structures methods suitable for supercell calculations, where noncollinear treatment of magnetism is implemented, have no means to locally constrain the axis along which the spin density matrix is diagonal, i.e., the direction of the local moments. In such cases, the control of the spin correlation functions of the supercell, the foundation of the scheme presented here, is lost. Furthermore, calculations of noncollinear magnetic systems are rather time consuming, and we leave the first-principles investigation of explicit effects of noncollinearity in disordered magnets to future studies.

\section{EFFECT OF STRONG ELECTRON CORRELATIONS}

Having established methods to treat the magnetic disorder within a supercell framework we now turn to the problem of electron exchange-correlation energies. Even though LDA calculations qualitatively revealed the energetics of $\mathrm{CrN}$ (Refs. 13 and 14) the electronic structure did not reproduce the experimentally observed semiconducting behavior. Thus one could doubt the accuracy of LDA predictions of structural and magnetic energy differences of relevance for understanding the orthorhombic to cubic transition in this system. Recently Herwadkar et al. studied CrN using the LDA $+U$ approach with focus on the electronic structure of ordered magnetic structures. They calculated the value of $U$ and $J$, the screened Coulomb and exchange terms, respectively, using constrained LDA approaches and achieved $U=3 \mathrm{eV}$ and $J=0.9 \mathrm{eV} .{ }^{15}$ However, they suggested a span of $U$ values from 3-5 eV to be reasonable. Even though such an $a b$ initio approach to obtain the values of the $U$ and $J$ parameters are appealing, the uncertainties are to large for a quantitative a thermodynamic analysis. Bhobe et al. ${ }^{12}$ attempted to measure the value of $U$ by means of resonant photoemission and estimated it to be $\sim 4.5 \mathrm{eV}$ both below and above the transition temperature.

In order to obtain the most suitable value $U^{e f f}$, for which the $\mathrm{LDA}+U$ method best describes the properties of $\mathrm{CrN}$ of relevance for this work, we perform a careful comparison of structural parameters and electronic structure obtained with $\mathrm{LDA}+U$ calculations for various values of $U$ with experiments. For comparison also the results obtained with the generalized gradient approximation, GGA, are presented.

First the lattice parameter of the cubic paramagnetic phase, modeled with the SQS approach, is presented in Fig. 3. The experimental value obtained for bulk $\mathrm{CrN}$ by Corliss et al. (Ref. 7) is $4.13 \AA$, while both Herle et al. ${ }^{44}$ and more recently, Rivadulla et al. ${ }^{8}$ obtained $4.148 \AA$. Values obtained 


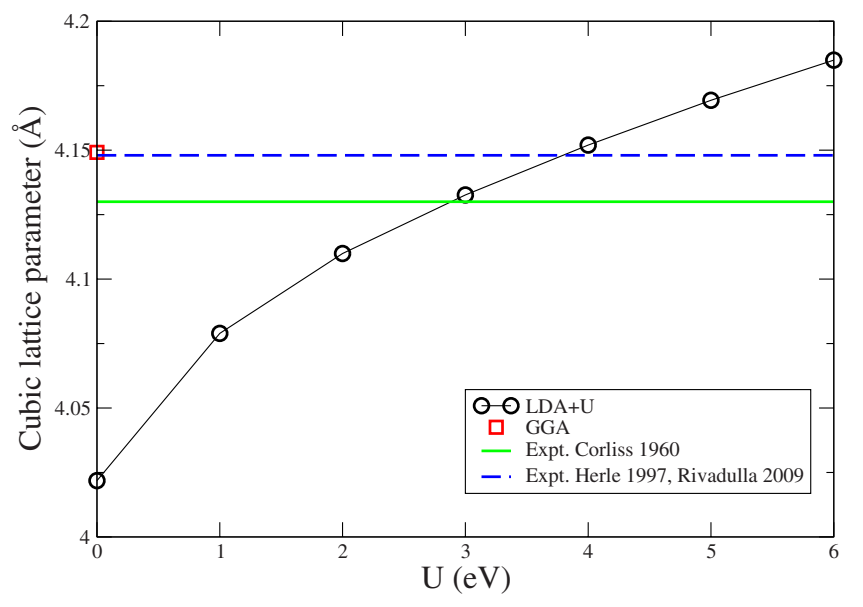

FIG. 3. (Color online) The calculated cubic lattice parameter for the magnetically disordered state within the LDA $+U$ (circles) and GGA (square) approximations. The experimental bulk value found by Corliss et al. (Ref. 7) and the common value found by Herle et al. (Ref. 44) and Rivadulla et al. (Ref. 8), are shown with solid and dashed horizontal lines, respectively.

for $\mathrm{CrN}$ in thin films are typically slightly larger ${ }^{9,10}$ but include strain effects not considered in the calculations. The result obtained with the pure LDA functional is $a$ $=4.022 \AA$. Using GGA we obtain $a=4.149 \AA$. The LDA $+U$ approach gives increasing lattice spacing with increasing $U$. The lattice parameter of Corliss et al. is obtained with $U=2.9 \mathrm{eV}$. The value of Herle et al. and Rivadulla et al. is obtained with $U=3.8 \mathrm{eV}$. Since the reported experimental values are measured at room temperature while the calculations with the exception for the magnetic disorder, corresponds to a $0 \mathrm{~K}$ situation, one might object that the comparison is not completely fair. However, thermal expansion between $0 \mathrm{~K}$ and room temperature is typically small in this class of hard ceramics.

Since also the physics of the orthorhombic phase must be well described by our theoretical model, we compare in Fig. 4 the calculated value of the angle $\alpha$ with the experimental finding in Refs. 7 and 8. $\alpha$ describes the angle between the axis of the conventional unit cell of the cubic B1 lattice, that is distorted in the orthorhombic phase, see the inset in Fig. 4. The experimental value is $88.3^{\circ}$ according to Corliss et al. ${ }^{7}$ or $88.4^{\circ}$ according to Rivadulla et al. ${ }^{8}$ while both LDA and GGA calculations underestimate this angle, thus overestimating the distortion. On the other hand, as can be seen in Fig. 4, a value in agreement with the experiment is obtained with the $\mathrm{LDA}+U$ method for $U=3.0 \mathrm{eV}$.

Finally, in Fig. 5 we compare the calculated total electronic density of states of the valence band of the disordered magnetic state (calculated with the SQS method) with the ultraviolet photoemission spectroscopy measurement of the cubic paramagnetic phase obtained by Gall et al. ${ }^{9}$ Figure 5 shows in different panels (from top to bottom) the density of states obtained with GGA, and $\mathrm{LDA}+U$ with $U$ from $0 \mathrm{eV}$ (LDA) to $U=5 \mathrm{eV}$. In all panels the experimental results are shown with dashed lines. In all cases we use the lattice spacing corresponding to the equilibrium of the particular choice of exchange-correlation scheme. The GGA, and even more

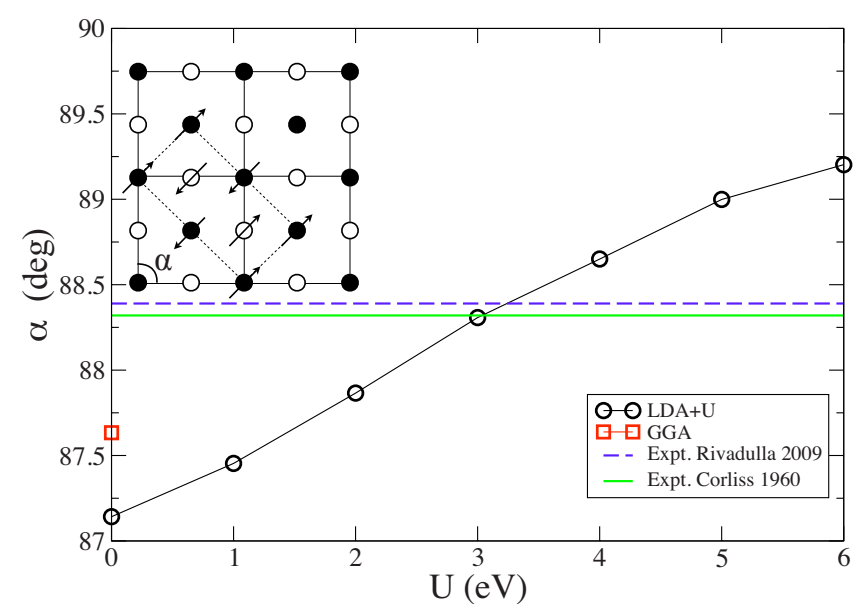

FIG. 4. (Color online) The calculated angle $\alpha$ in the distorted orthorhombic state between the axes of the conventional B1-cell. Values using the LDA $+U$ approximation (circles) and the GGA approximation (square) are shown together with the experimental value found by Corliss et al. (Ref. 7) (solid horizontal line) and Rivadulla et al. (Ref. 8) (dashed horizontal line). Inset: the definition of the angle $\alpha$ with respect to the orthorhombic unit cell (dashed lines) shown in a 001 plane of the cubic B1 structure. The relative directions of the spins of $\mathrm{Cr}$ atoms following Ref. 7 are also shown (solid circles: $z=0.0$ and open circles: $z=0.5$ ).

so the LDA, gives an overlap of the peaks close to the Fermi level. These peaks correspond primary to $\mathrm{Cr}$ spin-up nonbonding (below $E_{F}$ ) and a combination of spin down Cr non-

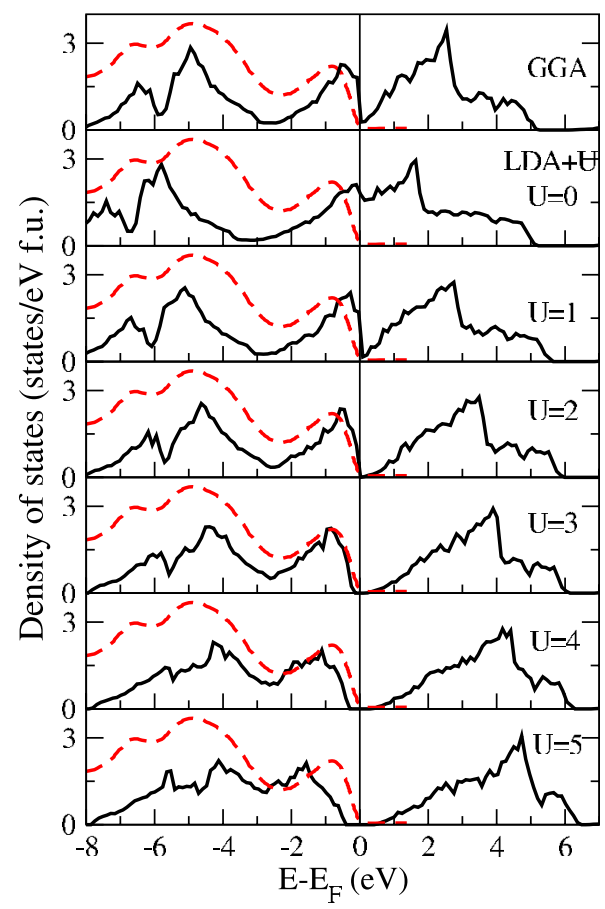

FIG. 5. (Color online) The calculated valence-band electronic density of states (solid line in all frames) of the cubic magnetically disordered state using the GGA approximation (top panel) and $\mathrm{LDA}+U$ approximation with different values of $U$. For comparison the experimental ultraviolet photoemission spectroscopy measurement by Gall et al. (Ref. 9) is shown by a dashed line in all frames. 


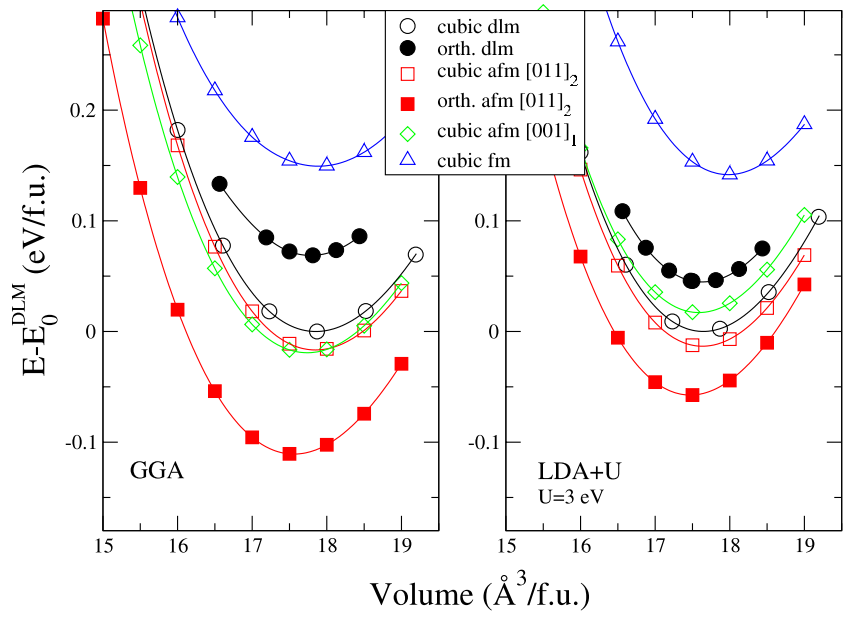

FIG. 6. (Color online) The calculated energy versus volume curves for different magnetic states in $\mathrm{CrN}$ using the GGA approximation (left panel) and LDA $+U(U=3 \mathrm{eV})$ (right panel). The energy minimum of the cubic collinear disordered DLM is used as reference energy.

bonding and $\mathrm{Cr}$ spin-up antibonding states (above $E_{F}$ ). Both peaks also have small admixture of $\mathrm{N} p$ character. ${ }^{15}$ When the $U$ value in the LDA $+U$ approach is increased, the occupied $\mathrm{Cr}$ spin-up nonbonding state becomes more localized and shift down in energy. At the same time the unoccupied states are shifted up and at a value of $U$ between 2 and $3 \mathrm{eV}$ a small gap opens at $E_{F}$, in agreement with the experiment. ${ }^{9}$ Actually the $\mathrm{LDA}+U$ approximation with $U=3 \mathrm{eV}$ excellently describes the $\mathrm{Cr}$ spin up nonbonding state while the bonding states on the other hand are shifted to slightly too high energies.

In summary, the LDA $+U$ approximation with $U$ values between about 3 and $4 \mathrm{eV}$ reproduces the cubic paramagnetic lattice parameter, the value $U=3 \mathrm{eV}$ reproduces experimental measurement of the angle $\alpha$ in the orthorhombic phase, and a value of $U$ between 2 and $3 \mathrm{eV}$ gives a good description of the electronic structure of the valence band of the cubic paramagnetic phase. Thus we conclude that within the $\mathrm{LDA}+U$ approximation, the value $U=3 \mathrm{eV}$ gives an optimal description of the physical properties of the system. This value is safely within the range suggested in Ref. 15 corresponding to $U=3.9$ if their choice of $J=0.9$ is used, and not to far away from the experimental estimate in Ref. 12.

In the following sections we use the $\mathrm{LDA}+U$ approximation with effective $U=3 \mathrm{eV}$ in our calculations. However, since the GGA approximation has already been used in many studies on related systems, we present also results using the GGA to illustrate the effect of strong electron correlations.

\section{ENERGETICS OF MAGNETIC AND CRYSTALLOGRAPHIC PHASES OF CrN}

We now have the theoretical tools needed to study the magnetostructural transition in CrN. In Fig. 6 we consider the total energies, as a function of volume of different crystallographic and magnetic phases of $\mathrm{CrN}$. The left panel shows the results obtained with the GGA functional while the right panel shows the results obtained with $\mathrm{LDA}+U(U$ $=3 \mathrm{eV}$ ). Cubic phases are shown with open symbols while orthorhombic (orth.) structures are shown with solid symbols. The paramagnetic cubic phase is modeled with the SQS disordered local moments method (denoted cubic dlm) keeping the atoms fixed at B1 lattice points. The energy of a disordered magnetic configuration on the lattice points of the orthorhombic structure is also shown for comparison and denoted orth. dlm. The minimum energy of the cubic collinear disordered magnetic phase is taken as the reference value. Furthermore, the energy of the experimental ground state structure, ${ }^{7}$ the orthorhombic distorted double [011] layered antiferromagnetic $\left([011]_{2} \mathrm{afm}\right)$ structure schematically shown in the inset of Fig. 4, is shown as is the energy of the same magnetic ordering on the cubic lattice. Also a single layer [001]-ordered antiferromagnetic state $\left([001]_{1} \mathrm{afm}\right)$ on the cubic lattice and the ferromagnetic cubic phase are shown.

One can see that the GGA and $\mathrm{LDA}+U$ calculations are in reasonable qualitative agreement with each other. However, the GGA gives considerably larger energy differences between the orthorhombic and cubic phases as compared to the LDA $+U$ calculations. Also the order of the two considered antiferromagnetic states for the cubic phase are reversed. In the GGA framework the $[001]_{1}$ antiferromagnetic state is lower in energy, in line with previous works. ${ }^{13,19}$ In the $L D A+U$ framework on the other hand, the $[011]_{2}$ antiferromagnetic state is lowest in energy also in the cubic phase.

One interesting comparison can be made between the introduction of a Hubbard $U$ term in this work and the alloying of $\mathrm{CrN}$ with AlN studied in Ref. 19. In that work it was found that the DLM-CPA state became lower in energy as compared to the $[001]_{1}$ antiferromagnetic state when a certain amount of $\mathrm{Al}$ was substituted for $\mathrm{Cr}$. The point is that upon alloying of $\mathrm{CrN}$ with $\mathrm{AlN},{ }^{19}$ and more generally upon alloying transition metal nitrides with $\mathrm{AlN},{ }^{45}$ the inclusion of $\mathrm{Al}$ favors a localization of the transition metal nonbonding $d$ states. Obviously, the strong electron correlations lead to a similar effect which explains the similar evolution of the magnetic energies in the two cases.

\section{MAGNETOSTRUCTURAL TRANSITION IN CrN}

Qualitatively our calculated values agree with the experimental observation of the stability of the orthorhombic state at low temperatures and a cubic state at higher temperatures. In this work we denote the temperature for this structural transition $T_{S}$ in order not to confuse it with the hypothetical isostructural Néel temperatures, $T_{N}$, of a magnetic orderdisorder transition on a fixed lattice. This is so since the disordered paramagnetic phase has a considerable magnetic entropy making it more competitive at higher temperatures. Since the energy of the cubic dlm state is considerably lower than the orthorhombic $\mathrm{dlm}$ state the magnetic disordering is accompanied by a structural transition. The fact that no signs of magnetic ordering was observed in the epitaxially stabilized cubic phases in Ref. 9 and 10 can be understood from the fact the energy differences between antiferromagnetic 
and disordered magnetic phases in the cubic geometry are small, indicating a very low Néel temperature for undistorted cubic $\mathrm{CrN}$. In the orthorhombic structure on the other hand the difference is almost an order of magnitude larger indicating that the orthorhombic antiferromagnetic state should be well below its isostructural Néel temperature at the experimental transition point $T_{S}^{\text {expt }}=280-287 \mathrm{~K} .^{7,8}$ This result is in line with the experimental observation that the extent of the orthorhombic distortion, measured with the value of the angle $\alpha$, is, in principle, the same at $286 \mathrm{~K}: 88.4^{\circ}, 8273 \mathrm{~K}$ : $88.3^{\circ},{ }^{46}$ and $77 \mathrm{~K}: 88.3^{\circ},{ }^{7}$ where the authors of the latter reference stated that no differences was seen when the temperature where further decreased down to the liquid-helium regime. If there had been a large degree of partial magnetic disorder in the orthorhombic phase one would expect a change in the value of this angle. Thus it is reasonable to assume that the transition is governed by the competition in terms of free energy between a disordered paramagnetic cubic phase with high magnetic entropy and a highly ordered antiferromagnetic orthorhombic phase with low magnetic entropy. Such a phase transition, including an abrupt change in both energy and entropy is in line with the experimental finding of a first-order phase transition displaying a hysteresis behavior during heating-cooling cycles. ${ }^{46}$

Using our obtained structural energy differences we can estimate the transition temperature theoretically. At temperatures considerably above the (isostructural) Néel temperature, such as in the case of paramagnetic $\mathrm{CrN}$ at room temperature, the entropy of a system with local moments can be approximated by the mean-field term

$$
S^{m f}=k_{B} \ln (M+1),
$$

where $M$ is the magnitude of the magnetic moment (in units of $\mu_{B}$ ) and $k_{B}$ is the Boltzmann constant. In the LDA $+U$ approach we find that the average magnetic moments are $M^{\mathrm{LDA}+U}=2.82 \mu_{B}$. In the GGA calculation $M^{\mathrm{GGA}}=2.49 \mu_{B}$.

Using these values and the approximations above the transition temperature can be obtained from the condition that at the critical temperature, the two phases should have the same free energy, $F$

$$
F_{\text {afm }}^{\text {orth }}\left(T_{S}\right)=F_{\text {para }}^{c u b}\left(T_{S}\right) \Leftrightarrow E_{\text {afm }}^{\text {orth }}=E_{\text {para }}^{c u b}-T_{S} S^{m f},
$$

where $T_{S}$ denotes the critical temperature for the structural transition which is in reality also the magnetic ordering temperature. In our case we get $T_{S}^{\mathrm{LDA}+U}=498 \mathrm{~K}$ using LDA $+U$ and $T_{S}^{\mathrm{GGA}}=1030 \mathrm{~K}$ using the GGA calculation. This mean-field estimates should be compared to the experimental value of $T_{S}^{\text {expt }}=280-287 \mathrm{~K}^{7,8}$ It is well known that the mean-field approximation, in general, overestimates magnetic ordering temperatures by as much as $50 \%$ as compared to more reliable thermodynamics treatments. However, in the GGA calculation the error is so large that we instead interpret this result as one more argument that the GGA approximation overemphasizes the orthorhombic distortion, both in geometric distortions visible in Fig. 4, and in the energy differences between the orthorhombic and cubic phases. The transition temperature derived from the $\mathrm{LDA}+U$ calculation is closer but still considerably above the experimental measurement, giving an overestimation of $T_{S}$ with $74 \%$. We sug-

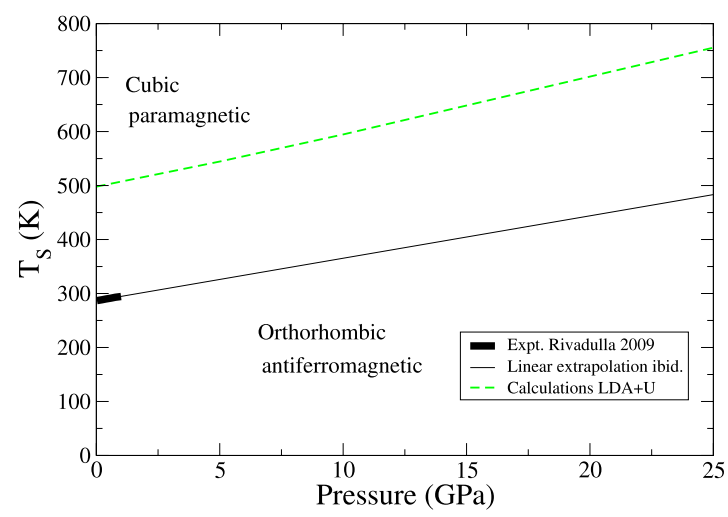

FIG. 7. (Color online) The calculated pressure-temperature phase diagram of $\mathrm{CrN}$ using the $\mathrm{LDA}+U(U=3 \mathrm{eV})$ method and considering the cubic paramagnetic phase as a disordered magnetic state. The experimental measurements of Rivadulla et al. (Ref. 8) at low pressures are shown with a bold black line while a linear extrapolation to higher pressures is shown with a thin black line.

gest that magnetic short-range order coupled with the vibrational degree of freedom is of importance to quantitatively determine the transition temperature in $\mathrm{CrN}$. Other possibilities that should be considered are that nitrogen offstoichiometry or explicit effects of noncollinear magnetism could influence the transition temperature. Moreover, one should remember that the $\mathrm{LDA}+U$ approach is an approximate method not free from errors, for instance, in the exact choice of $U$, possibly affecting quantitative values of the important structural energy difference.

Recently Rivadulla et al. ${ }^{8}$ showed that the temperatureinduced orthorhombic to cubic phase transition could be reversed with increasing pressure. At room temperature, a pressure as low as 1-2 GPa was enough to push the system back into the orthorhombic structure. Magnetic measurements showed that it was the antiferromagnetic ordered structure that reappeared. ${ }^{8}$ Qualitatively the pressure effect can be understood from the results in Fig. 6: since the orthorhombic phase is slightly lower in volume as compared to the paramagnetic cubic phase, it will be relatively more favorable at elevated pressures according to the minimization of the Gibb's Free energy

$$
G(T, p)=E+p V-T S .
$$

The derived pressure-temperature phase diagram of $\mathrm{CrN}$ is shown in Fig. 7. The results from the theoretical calculations using Eq. (7) with the mean-field approximation for the magnetic entropy of the cubic phase, Eq. (5), and the LDA $+U$ approximation for exchange-correlation effects are compared to the experimental low-pressure results from Ref. 8 and a linear extrapolation of these values to medium pressures. The qualitative picture, with increasing transition temperature with increasing pressure is rather well reproduced by the calculations although the absolute values of the temperatures are too high.

These results inspire us to propose a possible way to conclude the discussion of the value of the bulk modulus of cubic CrN. ${ }^{8,41,43}$ We suggest that in order to measure the 
compressibility of the cubic paramagnetic phase of $\mathrm{CrN}$ with higher accuracy as compared to Ref. 8, the experiment should be conducted at slightly higher temperatures where the cubic phase is stable over a larger pressure range.

\section{CONCLUSIONS}

We have used two different supercell approaches to model disordered magnetism of paramagnetic materials, the special quasirandom structure method and the magnetic sampling method, and applied them for the study of CrN. The SQS and MSM methods are shown to give equivalent results for calculations of disordered local moments on a fixed B1 lattice in cubic $\mathrm{CrN}$ and both of them agree with DLM-CPA calculations. We show that it is straightforward to extend the MSM method to calculations of noncollinear disordered magnetism.

$\mathrm{CrN}$ is a correlated material which is better described with an LDA $+U$ approach then with the GGA or LDA functionals. By comparing the calculated structural parameters and electronic structure of $\mathrm{CrN}$ with experiments we find that $U^{e f f}=3 \mathrm{eV}$ is a suitable value to use in the simulations.

Considering both magnetic disorder effects and strong electron correlations, the orthorhombic to cubic phase tran- sition of $\mathrm{CrN}$ as a function of temperature and pressure can be qualitatively explained. In particular, we show that it should be understood as a transition from a magnetically ordered orthorhombic phase to a magnetically disordered cubic phase. Considering magnetic entropy within the mean field approximation, the calculated transition temperature $T_{S}=498 \mathrm{~K}$ is an overestimation of the experimental value 280-287 K. Magnetic short-range order coupled with vibrational effects are likely to be of importance for determining the quantitative value of $T_{S}$. Since the transition also depends sensitively on the structural energy difference between the cubic and orthorhombic phases, which is shown to be very sensitive to the exchange-correlation functional, a strong electron correlations method beyond the $\mathrm{LDA}+U$ approach might be needed to reveal the details of the $\mathrm{CrN}$ phase transition.

\section{ACKNOWLEDGMENTS}

The Swedish Research Council (VR), the Swedish Foundation for Strategic Research (SSF), and the Göran Gustafsson Foundation for Research in Natural Sciences and Medicine are acknowledged for financial support. Calculations were performed using computational resources allocated by the Swedish National Infrastructure for Computing (SNIC). *bjoal@ifm.liu.se

${ }^{1}$ H. Holleck, J. Vac. Sci. Technol. A 4, 2661 (1986).

${ }^{2}$ O. Knotek, M. Atzor, A. Barimani, and F. Jungblut, Surf. Coat. Technol. 42, 21 (1990).

${ }^{3}$ R. Wuhrer and W. Yeung, Scr. Mater. 50, 1461 (2004).

${ }^{4}$ A. E. Reiter, V. H. Derflinger, B. Hanselmann, T. Bachmann, and B. Sartory, Surf. Coat. Technol. 200, 2114 (2005).

${ }^{5}$ J. Vetter, Surf. Coat. Technol. 76, 719 (1995).

${ }^{6}$ A. Persson, J. Bergström, C. Burman, and S. Hogmark, Surf. Coat. Technol. 146, 42 (2001).

${ }^{7}$ L. M. Corliss, N. Elliott, and J. M. Hastings, Phys. Rev. 117, 929 (1960).

${ }^{8}$ F. Rivadulla et al., Nature Mater. 8, 947 (2009).

${ }^{9}$ D. Gall, C. S. Shin, R. T. H. I. Petrov, and J. E. Greene, J. Appl. Phys. 91, 5882 (2002).

${ }^{10}$ R. Sanjinés, O. Banakh, C. Rojas, P. E. Schmid, and F. Lévy, Thin Solid Films 420-421, 312 (2002).

${ }^{11}$ X. Y. Zhang and D. Gall, Phys. Rev. B 82, 045116 (2010).

${ }^{12}$ P. A. Bhobe et al., Phys. Rev. Lett. 104, 236404 (2010).

${ }^{13}$ A. Filippetti, W. E. Pickett, and B. M. Klein, Phys. Rev. B 59, 7043 (1999).

${ }^{14}$ A. Filippetti and N. A. Hill, Phys. Rev. Lett. 85, 5166 (2000).

${ }^{15}$ A. Herwadkar and W. R. L. Lambrecht, Phys. Rev. B 79, 035125 (2009).

${ }^{16}$ P. Olsson, I. A. Abrikosov, L. Vitos, and J. Wallenius, J. Nucl. Mater. 321, 84 (2003).

${ }^{17}$ B. L. Gyorffy, A. J. Pindor, J. Staunton, G. M. Stocks, and H. Winter, J. Phys. F: Met. Phys. 15, 1337 (1985).

${ }^{18}$ P. Soven, Phys. Rev. 156, 809 (1967).

${ }^{19}$ B. Alling, T. Marten, I. A. Abrikosov, and A. Karimi, J. Appl.
Phys. 102, 044314 (2007).

${ }^{20}$ I. A. Abrikosov, A. E. Kissavos, F. Liot, B. Alling, S. I. Simak, O. Peil, and A. V. Ruban, Phys. Rev. B 76, 014434 (2007).

${ }^{21}$ F. Liot and I. A. Abrikosov, Phys. Rev. B 79, 014202 (2009).

${ }^{22}$ A. Zunger, S. H. Wei, L. G. Ferreira, and J. E. Bernard, Phys. Rev. Lett. 65, 353 (1990).

${ }^{23}$ P. E. Blöchl, Phys. Rev. B 50, 17953 (1994).

${ }^{24}$ G. Kresse and J. Hafner, Phys. Rev. B 48, 13115 (1993).

${ }^{25}$ G. Kresse and D. Joubert, Phys. Rev. B 59, 1758 (1999).

${ }^{26}$ D. M. Ceperley and B. J. Alder, Phys. Rev. Lett. 45, 566 (1980).

${ }^{27}$ J. P. Perdew, K. Burke, and M. Ernzerhof, Phys. Rev. Lett. 77, 3865 (1996).

${ }^{28}$ V. I. Anisimov, J. Zaanen, and O. K. Andersen, Phys. Rev. B 44, 943 (1991).

${ }^{29}$ S. L. Dudarev, G. A. Botton, S. Y. Savrasov, C. J. Humphreys, and A. P. Sutton, Phys. Rev. B 57, 1505 (1998).

${ }^{30}$ H. J. Monkhorst and J. D. Pack, Phys. Rev. B 13, 5188 (1976).

${ }^{31}$ L. Vitos, J. Kollár, and H. L. Skriver, Phys. Rev. B 55, 13521 (1997).

${ }^{32}$ L. Vitos, I. A. Abrikosov, and B. Johansson, Phys. Rev. Lett. 87, 156401 (2001).

${ }^{33}$ L. Vitos, Phys. Rev. B 64, 014107 (2001).

${ }^{34}$ A. M. N. Niklasson, J. M. Wills, M. I. Katsnelson, I. A. Abrikosov, O. Eriksson, and B. Johansson, Phys. Rev. B 67, 235105 (2003).

${ }^{35}$ A. E. Kissavos, S. I. Simak, P. Olsson, L. Vitos, and I. A. Abrikosov, Comput. Mater. Sci. 35, 1 (2006).

${ }^{36}$ P. A. Korzhavyi, A. V. Ruban, J. Odqvist, J.-O. Nilsson, and B. Johansson, Phys. Rev. B 79, 054202 (2009).

${ }^{37}$ B. Alling, A. V. Ruban, and I. A. Abrikosov, Phys. Rev. B 79, 
134417 (2009).

${ }^{38}$ V. Heine, A. I. Liechtenstein, and O. N. Mryasov, Europhys. Lett. 12, 545 (1990).

${ }^{39}$ A. V. Ruban and I. A. Abrikosov, Rep. Prog. Phys. 71, 046501 (2008).

${ }^{40}$ B. Alling, A. V. Ruban, A. Karimi, O. E. Peil, S. I. Simak, L. Hultman, and I. A. Abrikosov, Phys. Rev. B 75, 045123 (2007).

${ }^{41}$ B. Alling, T. Marten, and I. A. Abrikosov, Nature Mater. 9, 283 (2010).
${ }^{42}$ B. Alling, Phys. Rev. B 82, 054408 (2010).

${ }^{43}$ F. Rivadulla et al., Nature Mater. 9, 284 (2010).

${ }^{44}$ P. S. Herle, M. S. Hegde, N. Y. Vasathacharya, S. Philip, M. V. R. Rao, and T. Sripathi, J. Solid State Chem. 134, 120 (1997).

${ }^{45}$ B. Alling, A. Karimi, and I. A. Abrikosov, Surf. Coat. Technol. 203, 883 (2008).

${ }^{46}$ J. D. Browne, P. R. Liddell, R. Street, and T. Mills, Phys. Status Solidi A 1, 715 (1970) 